\title{
The Test and Vector Extension Model of the Vessel Static Electric Field Xiangjun Wang $^{1,}$, , Fang $\mathrm{He}^{1 \text {, a }}$ \\ ${ }^{1}$ Electrical Engineering College, Naval University of Engineering, Wuhan, China, 430033 \\ a Wskay1103@163.com
}

Keywords: vector continuation; static electric field; the semi infinite domain

Abstract: The continuation of the static electric field is based on the electric field scalar potential or the three component of the electric field, and the calculation process is complex. According to the vector Green formula, the static electric field vector continuation model is established based on the integral equation for simplifying the calculation. The unity of this model and the infinite plane method is proved. Because the static electric field vector continuation model replaces the infinite plane with the closed surface, it can be used to solve the problem of calculating efficiency of the infinite plane method. Simulation results demonstrate the feasibility of the model. The test results show that the relative error of the continued and the measured is about $5 \%$.

\section{Introduction}

The static electric field has three characteristics ${ }^{[1-2]}$ : (1) It can not be avoided in seawater. (2) It spread far. If the frequency is to zero, the field can spread far away. (3) It is difficult to be measured and detected. Because static electric field signal is completely submerged in seawater noise, it is difficult to separate; at the same time, various types of electrostatic field signal propagation characteristics is not obvious, so it is difficult to detect. To master the distribution of far field, it relates to the conversion of static electric field. At present, there is some method ${ }^{[3-6]}$, as boundary element method (BEM), finite element method (FEM), equivalent electric dipole method, point power method, iteration method, and infinite plane method. These methods are generally based on the scalar potential of the electric field or the three component of the electric field, and the calculation is complex. Static electric field vector extension model from the electric field vector angle conversion and solution of scalar potential under water, the negative gradient method, can greatly simplify the process of calculation; and the model with a closed surface instead of the infinite plane, can well solve the problem of infinite plane method in measuring surface approximate infinite caused by computational efficiency decline.

\section{The Static Electric Field Vector Extension Model and the Infinite Plane Method}

A static electric field vector extension model can be described by formula $1^{[7]}$. By this model, the electric field distribution in the semi infinite passive domain can be solved greatly simplified without the demand of the solution of the scalar potential and then the negative gradient. The establishment of Cartesian coordinates in an infinite plane, as shown in figure 1. A source point, located in the source region, is located in the semi infinite passive region, and the $\mathrm{S}$ mirror image symmetry as the unit axial vector.
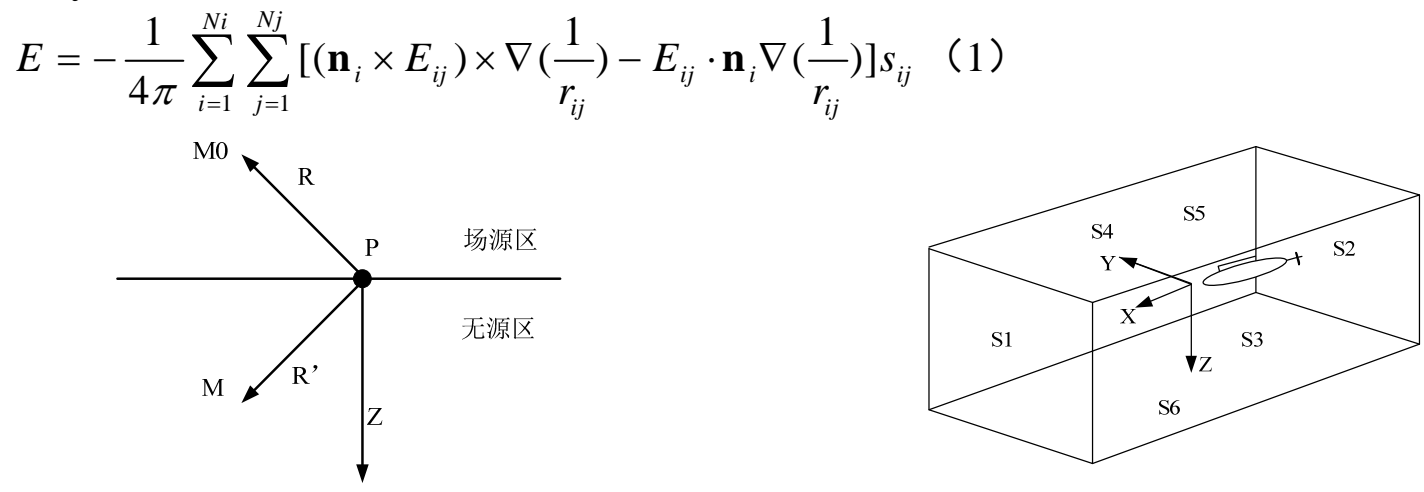

Fig 1.Schematic of mirror symmetry on the infinite plane

Fig 2.The Boundary surface around Field source 


$$
\nabla \frac{1}{R}=-\frac{1}{R^{3}}\left[\left(x-x_{P}\right) e_{x}+\left(y-y_{P}\right) e_{y}+\left(z-z_{P}\right) e_{z}\right] \nabla \frac{1}{R^{\prime}}=-\frac{1}{R^{\prime 3}}\left[\left(x-x_{P}\right) e_{x}+\left(y-y_{P}\right) e_{y}+\left(z+z_{P}\right) e_{z}\right]
$$

Formula 2 can describe the source region, and formula 3 can describe the no source region:

$$
E_{\mathrm{M} 0}\left(x_{0}, y_{0}, z_{0}\right)=\frac{1}{4 \pi} \int_{s}(\mathbf{n} \times E) \times \nabla\left(\frac{1}{R}\right) d s \frac{1}{4 \pi} \int_{s} E \cdot \mathbf{n} \nabla\left(\frac{1}{R}\right) d s \quad(2) \quad 0=\frac{1}{4 \pi} \int_{s}(\mathbf{n} \times E) \times \nabla\left(\frac{1}{r}\right) d s+\frac{1}{4 \pi} \int_{s} E \cdot \mathbf{n} \nabla\left(\frac{1}{r}\right) d s
$$

Formula 2 plus formula 3, formula 4 can be get:

$$
E=\frac{z_{0}}{2 \pi} \int_{s} \frac{E_{x} e_{x}+E_{y} e_{y}+E_{z} e_{z}}{R^{3}} d s=\frac{z_{0}}{2 \pi} \int_{s} \frac{E(x, y, z)}{R^{3}} d s
$$

Formula 4 has the same form as the theoretical formula of the infinite plane method of the literature [8]. When the closed curved surface is extended to the infinite plane, the static electric field vector extension model and the infinite plane method are unified. According to the literature [9], it can be known that the infinite plane method has strict requirements on the measurement surface. If the conversion of the target surface is close to the measurement surface, it is impossible to achieve a large error. If the scope of the measurement surface is enlarged, while the conversion accuracy is improved, the conversion efficiency will be reduced. The closed surface is used to replace the infinite plane in the static electric field vector extension model, which can solve the problem of the computation efficiency drop which is caused by the measurement surface approximation in the infinite plane method. Therefore, the static electric field vector extension model is unified with the infinite plane method, and is not limited to the "infinite plane ".

\section{The Feasibility and Error Analysis of Extension Model of Static Electric Field}

In order to verify the feasibility and accuracy of the underwater static electric field vector extension model, MATLAB is used for simulation. Approximate ellipsoid submarine can be equivalent to an electric dipole, a unit of electric dipole placed in the water depth of 5 , the design of a closed side interface for a rectangular in vitro surface, as shown in figure 2, length and width, respectively. In order to simulate the actual situation close to the actual situation (to facilitate the submarine through), without taking into account the electric field distribution of S1, S2 two sides, the remaining four sides of each face are divided into 648 square faces, a total of 2592 face. According to formula 4, the electric field vector of the arbitrary position in a semi infinite region outside the rectangular surface area is obtained.

2.1 feasibility validation. The static electric field vector extension model is extrapolated plane on the electric field distribution, as shown in figure 3.The simulation results were compared with the literature [9] , and the infinite plane method is then converted into the same plane of the electric field vector is completely consistent in magnitude.
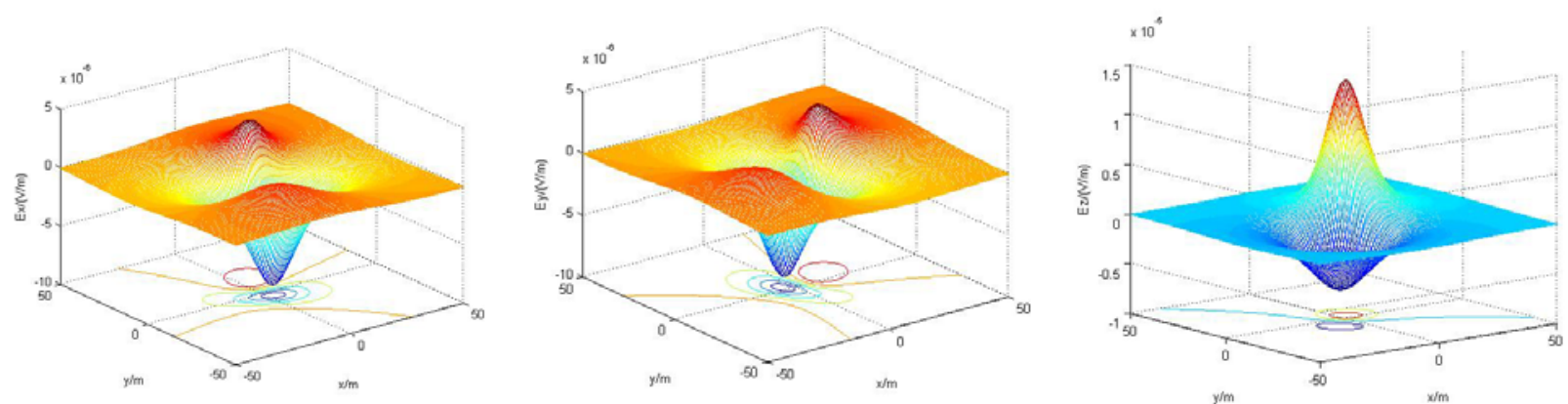

Fig 3.The results of continuation plane

The simulation results show that the static electric field vector extension model of underwater static electric field is correct, and it is proved that it is feasible and reliable to design the experimental design of the closed surface with rectangular four surfaces.

2.2 error analysis. Applied static electric field vector extrapolation model calculated line E1 electric field values; the literature [9] the infinite plane method, is approximated by the infinite plane, the conversion plane line E2 electric field values; the literature [10], the electric field distribution of analytical expressions for calculating the electric field on the straight line E3 value. 
Taking E3 as the theoretical reference value (E3 is equivalent to the dipole modeling formula, model validation results accuracy is high, as a static field modeling empirical formula), the results of the comparison of the three are shown in figure 4. The calculated values of E1, E2 and the theoretical value of E3 in the three component of the electric field are in complete agreement, but there is a certain amount of error in the numerical value. Compared to the E1 calculated by the static electric field vector extension model, the E2 deviation from the theoretical value of the infinite plane method is larger than that of the E3.
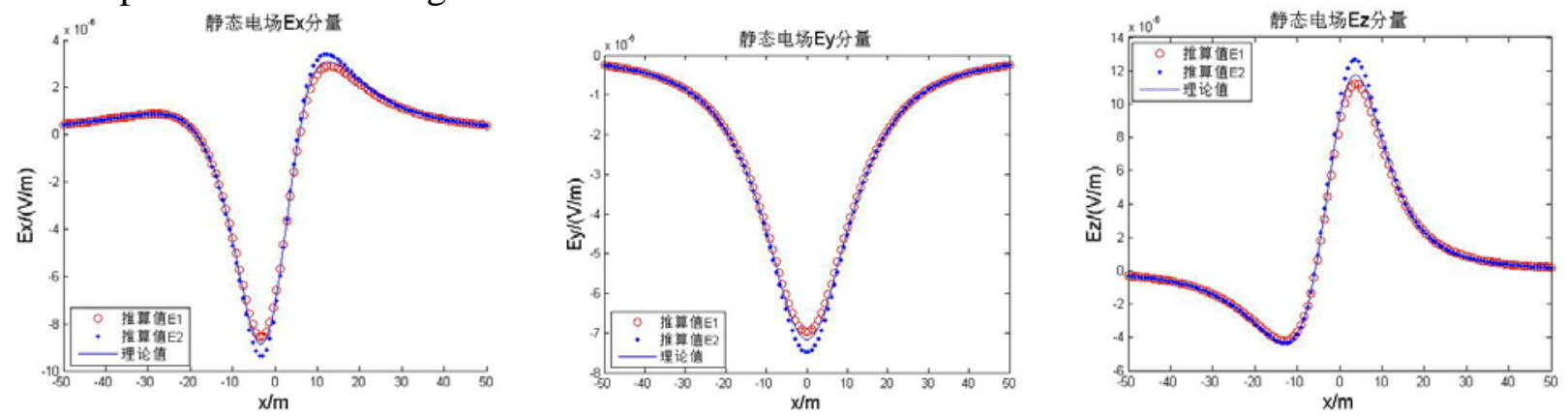

Fig 4.The results of the calculated and the theoretical

The main reason for the error is: (1) based on the actual measurement, the static electric field vector extension model is used to calculate the front and rear two end faces of the envelope surface. The simulation results show that the error is not small, but it still generates a certain error because of the small area of the front and back ends of the front and back. (2) in the vector integral surface meshes, each surface element is the center point of the electric field vector as the vector, will produce the discretization error. Therefore, the facet element mesh share more fine, the surface element more, the surface area is small, accurate projection will higher.

\section{The Experiment and Data Analysis of Static Electric Field Vector Extension Method}

Based on the static electric field vector extension theory model, the distribution of the static electric field is extrapolated. On the basis of simulation results, in order to further verify the effectiveness and accuracy of the method, the submarine model is used in the laboratory to verify the effectiveness of the proposed method.

3. 1 experimental condition. The experimental hardware facilities include a non magnetic experimental pool, an experimental submarine model, an electric field vector sensor and a rectangular measuring frame. Non magnetic experimental pool, size of $8 \mathrm{~m} * 5 \mathrm{~m} * 1.5 \mathrm{~m}$, water depth of $0.6 \mathrm{~m}$, the industrial salt into the pool water, fully dissolved and mixed evenly, simulated dilute brine, the measured water conductivity of $3.96 \mathrm{~S} / \mathrm{m}$. Submarine model is $1.5 \mathrm{~m}$ long, $0.13 \mathrm{~m}$ wide, $0.15 \mathrm{~m}$ high, according to the proportion of 1:50 to reduce the proportion of real craft. Rectangular measuring frame width $0.4 \mathrm{~m}$, high $0.4 \mathrm{M}$. Fully placed in the water fixed, measuring frame on each side of the uniform distribution of 3 electric field vector sensor.
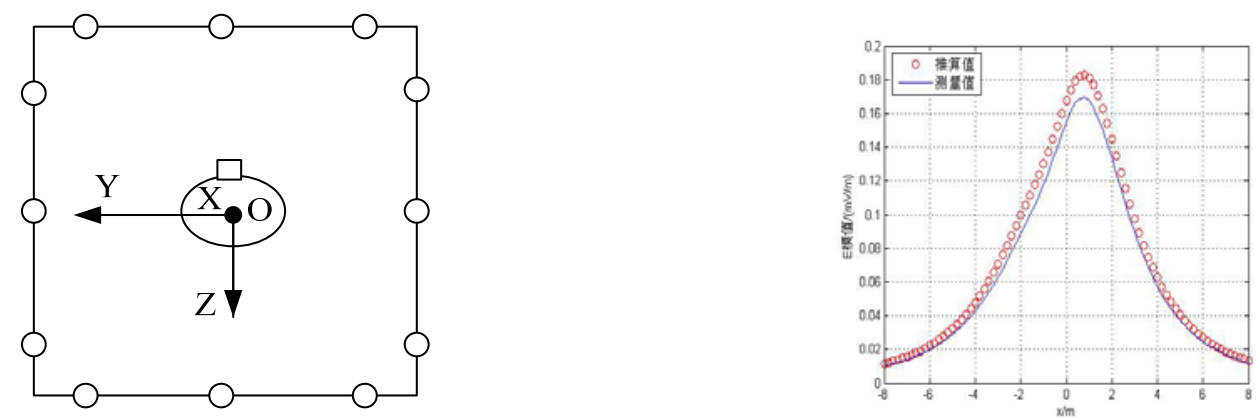

Fig 5.Schematic of position of submarine and sensor Fig 6. Comparision between the measured and computed

The experimental steps are as follows: (1) move the ship model to the distance measurement frame, the electric field vector sensor collects the background electric field. (2) to measure the frame as the center to establish a Cartesian coordinate system, the submarine along the $\mathrm{X}$ axis uniformly across the measurement frame, as shown in figure 5 (submarine from within the paper 
gypsum movement). Electric field vector sensor can collect the electric field value of boat models in each position, and then a rectangular envelope can be get in addition to the front and the end surface. Using the electric field at each measurement point on the rectangular envelope to subtract the background electric field, the electric field distribution on the rectangular envelope can be obtained. (3) according to the established static electric field vector extrapolation model estimated distance submarine port $3 \mathrm{~m}$, water depth of $0.2 \mathrm{~m}$, namely linear electric field. (4) the electric field distribution in the straight line, according to the mathematical relationship between the electric field model and the vector of the electric field, the electric field mode. The electric field mode value is calculated by the static electric field vector extension model, and compared with the field mode value of the measured line, the results are shown in figure 6.

3.2 expertimental data analysis. The ship model experiment in the electric field can be seen as the source point, straight line at various points as an example. At the center of the ship model for the axis of symmetry, in deviation from the source point is near the mode of the electric field value of large amplitude attenuation from point source distance decay becomes weak. Established in the Cartesian coordinates, ship model center coordinates $(0,0,0)$, far-field coordinates $(0,3,0.2)$ and $(6$, $3,0.2$ ) substituted [10] in static electric field distribution analytic formula is verified, can be found in Captain about 4 times and static electric field strength modulus attenuation of about $75 \%, 4$ times less decay faster, 4 times than decay slowly. It is shown that the experiment is effective in the experimental data and the literature [10] DC electric dipole equivalent theory formula at the same point source and far-field calculation results are consistent. According to equation (5) shows that the electric field intensity between the field point and the source point is inversely proportional to the distance. On the spot point and source point distance is close, electric field intensity decay faster; field point and the source point distance is far, the electric field intensity decay more slowly. The error between the conversion mode of the static electric field vector extension model and the measured electric field mode value is defined as the error between the measured electric field model, which is converted to the electric field modulus of the static electric field vector extension model. After analysis, the relative error of the electric field model is about $5 \%$, which can achieve higher conversion accuracy.

\section{Conclusions}

In this paper, a static electric field vector extension model is established on the basis of the integral equation, and the image method is used to prove the model and the infinite plane method. Simulation results show that the static electric field vector extension of the feasibility of the extension model; submarine model experimental results show that, the static electric field vector extension of model calculation mode of the electric field values and the measured electric field mode values of relative error is about 5\%. (1) the superiority of the model: no need to solving scalar potential, then the negative gradient solution directly from the angle of the electric field vector of underwater static electric field distribution calculation, greatly simplifies the calculation process, and the model is not limited to "infinite plane", to better solve the measurement surface approximate infinite caused by the computational efficiency is reduced. (2) the model error causes: Based on actual measurement is more convenient, this paper ignores enveloping surface before and after the two end faces, to approximate closed surface, due to the small area of the front and rear ends of the surface. The simulation results show that error is small, but can still create calculation errors. In the vector integral surface meshes, each surface element is the center point of the electric field vector as the vector, will produce the error. Therefore, the facet element mesh share more fine, surface element more, surface element of the area is small, calculated precision higher and higher.

\section{Acknowledgements}

This work was financially supported by the National Natural Science Foundation (41476153). 


\section{References}

[1] Lin Chunsheng,Gong Shenguang. Ship physical field (Second ed.) [M]. Beijing: Weapon Industry Press, 2007:23-25.[in Chinese]

[2] Gong Shenguang, Liu Shengdao et al. The study on the spatial distribution characteristics model and experimental of ship electric field caused by corrosion and corrosion protection [R]. Wuhan: Naval Engineering University, 2004. [in Chinese]

[3] ZAMANI N G. Boundary element simulation of the cathodic protection system in a prototype ship[J].Applied Mathematics and Computation,1988,26:119-123.

[4] IWATA M, HUANG Y, FU JIMOTO Y. Application o f BEM to design o $f$ the impressed current cathodic protection system for ship hull [J]. J of the Society of Naval Architects of Japan, 1992, 171: 377-380.

[5] DOIG P,FLFWITT P E J.A finite difference numerical analysis of galvanic corrosion [J],Electrochem,1979,126(12):2057-2063.

[6] KASPER R G, APRIL M G. Electrogalvanic finite element analysis o f partially protected marine structures [J]. Corrosion, 1983, 39(5): 181-183.

[7] He Fang, Wang Xiangjun, Gao Junji. Extrapolation of vessel static electric field[J].Journal of detection and control,2015,06: 55-60[in Chinese]

[8] Li Dingguo,Chen Cong,Liu Huabo. Creen formula method for extrapolating of ship’s underwater static electric field[J]. Naval University of Engineering, 2012, 24 (3) : 1-5. [in Chinese]

[9] Chen Cong,Li Dingguo,Gong Shenguang. Research on the continuation of the static electric field of ships based on Laplace Equation[J].Acta Electronica Sinica, 2010, 38(9) : 2025-2030. [in Chinese]

[10] Liu Shengdao. Research on Measuring Technique and Electric Dipole Model of Ship's Underwater Electric Field[D]. Wuhan: Naval University of Engineering, 2002: 43-45. [in Chinese] 